\title{
Archéopages
}

Archéopages

Archéologie et société

Hors-série 3 | 2012

Nouveaux champs de la recherche archéologique

\section{Climats et cultures paléolithiques : quand la vallée devient frontière...}

Laurent Bruxelles et Marc Jarry

\section{OpenEdition}

1 Journals

Édition électronique

URL : https://journals.openedition.org/archeopages/469

DOI : 10.4000/archeopages.469

ISSN : 2269-9872

Éditeur

INRAP - Institut national de recherches archéologiques préventives

Édition imprimée

Date de publication : 1 janvier 2012

Pagination : 72-85

ISSN : 1622-8545

Référence électronique

Laurent Bruxelles et Marc Jarry, «Climats et cultures paléolithiques : quand la vallée devient

frontière... », Archéopages [En ligne], Hors-série 3 | 2012, mis en ligne le 01 janvier 2012, consulté le 23

février 2023. URL : http://journals.openedition.org/archeopages/469; DOI : https://doi.org/10.4000/

archeopages. 469 
Climats et cultures paléolithiques : quand la vallée devient frontière...

Laurent Bruxelles

Inrap, UMR 5608 «Travaux et Recherches Archéologiques sur les Cultures, les Espaces et les Sociétés », Université de Johannesburg Marc Jarry

Inrap, UMR 5608 " Travaux et Recherches Archéologiques sur les Cultures, les Espaces et les Sociétés »

$\mathrm{E}$ n 2002, dans un article de synthèse concernant le Paléolithique de la région toulousaine, Jacques Jaubert évoquait l'absence de vestige du Paléolithique supérieur (Jaubert, 2002). Effectivement, alors que les témoins des phases anciennes du Paléolithique sont bien représentés, les plus récentes sont pour ainsi dire absentes. Pour expliquer ce constat, il avait proposé trois explications possibles. D'abord, un biais dans la recherche: celle-ci aurait été insuffisante ou les sites ne seraient pas accessibles du fait, par exemple, d'un important recouvrement sédimentaire; ensuite, la conservation différentielle: les terrains de cette période (et les sites qui vont avec) auraient été purgés par les phénomènes péri- et post-glaciaires; enfin, la rareté effective d'établissements de plein air au Paléolithique supérieur dans certains contextes morphologiques. C'est à cette question que nous nous proposons de répondre ici, dans la continuité des travaux engagés sur cette problématique, en nous appuyant sur les résultats de dix ans d'archéologie préventive dans la vallée de la Garonne (Bruxelles et al., 2003; Jarry, 2008; Bruxelles et al., 2010; Jarry 2010 ; Bruxelles, Jarry, 2011).

Historiographie des recherches. Dans un cadre plus large, les régions septentrionales de l'Europe ont connu, au cours du Pléistocène moyen et supérieur, des peuplements largement influencés par les fluctuations climatiques quaternaires (Otte, 1983; Gamble, 1986; Conard, 1992; Roebroeks et al., 1992 ; Tuffreau, 1992 ; Antoine et al., 2003). Nous n'entrerons pas ici dans le débat sur les modèles d'occupation pendant les interglaciaires (Gamble, 1986 ; Roebroeks et al., 1992 ; Antoine et al., 2003) pour nous focaliser sur les pléniglaciaires. Lors de ces périodes, il est maintenant admis qu' une désertion, plus ou moins marquée, par les populations préhistoriques (avec un hiatus complet de 23000 à 13000 avant notre ère pour le Weischelien), a touché ces régions.

Alors que le sud jouerait plutôt le rôle de «plateforme de peuplement» (Soriano, 2005), les zones méridionales et, parmi elles, les espaces aquitains, seraient alternativement des « refuges» ou des «réservoirs » pour les populations des espaces septentrionaux désertés pendant les péjorations climatiques. Nous resterons réservés sur le terme de refuge qui nous paraît difficile à envisager à cette échelle. Mais toujours est-il que le patrimoine archéologique du Sud-Ouest est tel que, pour toutes les périodes du Paléolithique, l'image communément admise est celle, comme pour le reste de la « corne aquitano-cantabrique », d'une région ayant connu une occupation humaine continue (Straus, 1991; Demars, 1996 et 2006 ; Bocquet-Appel, Demars, 2000a et 200ob; Gamble et al., 2004; BocquetAppel et al., 2005; Demars, 2008; Banks et al., 2006, 2008 et 2009). Ces approches récentes, considérant les grands ensembles culturels et les peuplements par la répartition et la densité des sites, ont une résolution telle qu'elles englobent, dans une même entité ou presque, l'ensemble du bassin aquitain.

Pour le Paléolithique supérieur, des études plus détaillées ont cependant permis d'approcher d'un peu plus près l'occupation de l'espace compris entre Massif Central et Pyrénées. Ont pu être perçus des mouvements de populations, rythmés par l'avancée et le recul des glaciers couronnant les massifs montagnards (Méroc, 1961; Clottes 1976; Demars, 1996, 2000, 2002a, 2002b, 2004 et 2008; Bocquet-Appel, Demars, 20ooa). Cette approche des fluctuations du peuplement des zones d'altitude est envisageable du fait de l'impossibilité d'occuper des espaces englacés lors des maximums glaciaires. Rappelons que les glaciers pyrénéens (cf. cartographie des glaciers pyrénéens par Andrieu et al., 1988, et Calvet, 2004), dont la période majeure d'extension se situe entre 30 ooo et 20000 avant notre ère, commencent leur retrait dès 23000 (Pallas et al., 2006). Ces langues glaciaires descendent alors très bas dans les vallées (par exemple, Foix et Lourdes sont alors sous les glaces).

Mais qu'en est-il de l'occupation des vallées et des plaines? Les sites du Paléolithique supérieur, toutes cultures confondues, sont très abondants entre Massif Central et Pyrénées, mais ils ne couvrent pas tout l'espace disponible. Les sites et les indices découverts au sud, dans le piémont pyrénéen, et au nord, dans les canyons des causses, sont très nombreux et indiquent une occupation pérenne dans ces secteurs géographiques intermédiaires. Par contre, les vestiges attribuables au Paléolithique supérieur sont aujourd'hui encore quasiment absents des grandes plaines du Midi toulousain. Ce phénomène, contrastant nettement avec les très nombreux vestiges retrouvés pour les phases anciennes du Paléolithique, est connu de longue date. Cependant, les implications restaient difficiles à développer, faute d'argument péremptoire sur la réalité de l'occupation. Quelle peut être la ou les raisons de cette «indigence»?

Après une présentation du contexte géomorphologique, nous nous attacherons à valider ou invalider chacune des hypothèses émises par J. Jaubert. Puis, nous nous proposons alors de réinterpréter l'occupation de cette entité géographique pendant le Paléolithique supérieur, c'est-à-dire pendant la fin du Dernier Interpléniglaciaire (fin $\mathrm{OIS}_{3}$ ) et la péjoration climatique du Dernier Glaciaire jusqu'au Tardiglaciaire (OIS 2). Cette problématique est essentielle, car des conditions de son peuplement dépendent le modèle d'occupation du «territoire» aquitain dans sa globalité, avec les implications culturelles qui peuvent en être attendues, comme cela a pu être proposé pour la vallée de la Garonne (Bruxelles, Jarry, 2011) ou pour les Landes de Gascogne (Bertran, à paraître). Il sera ensuite discuté, sur la base de ce modèle, de la réalité

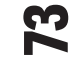



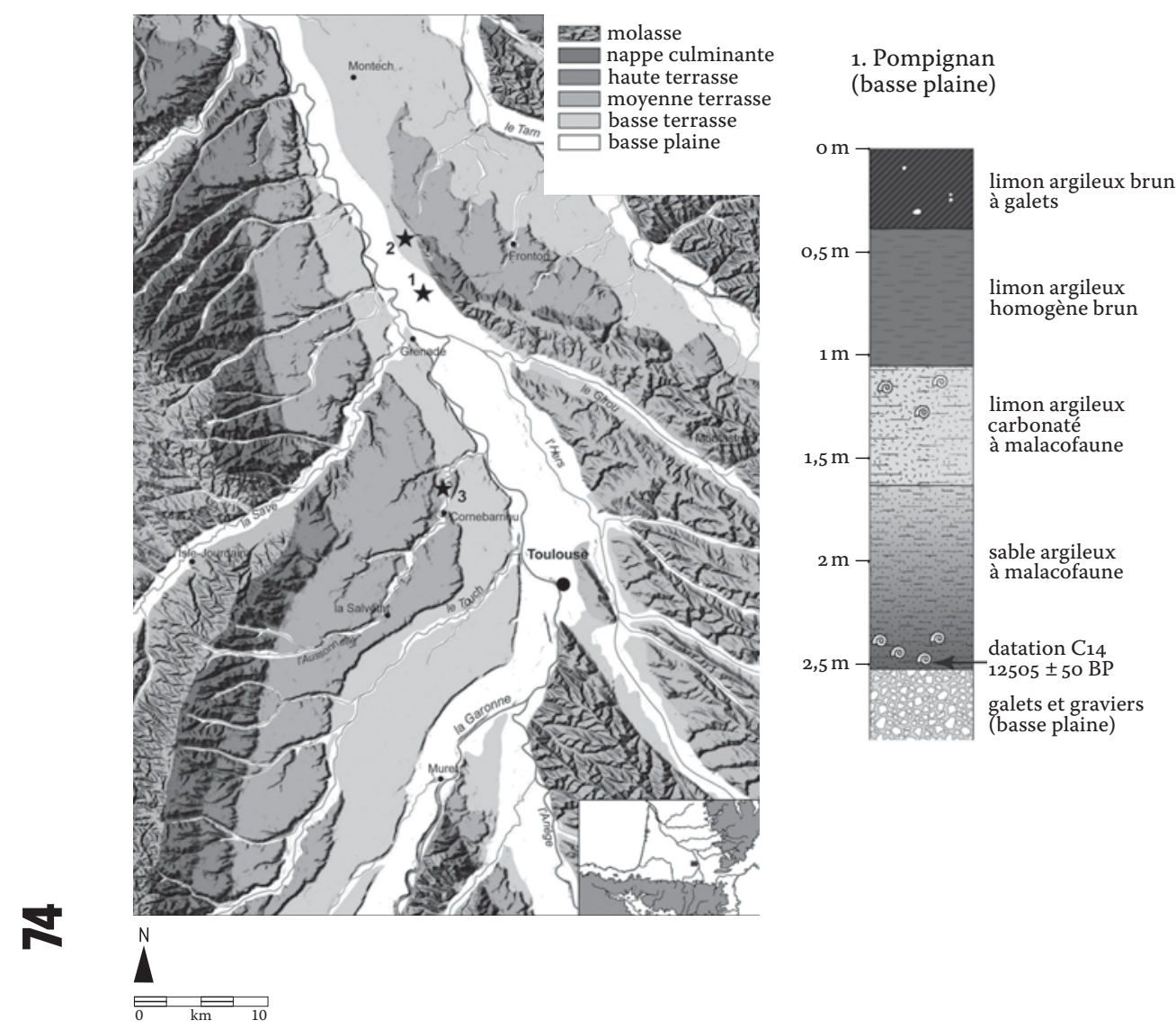

(basse terrasse)

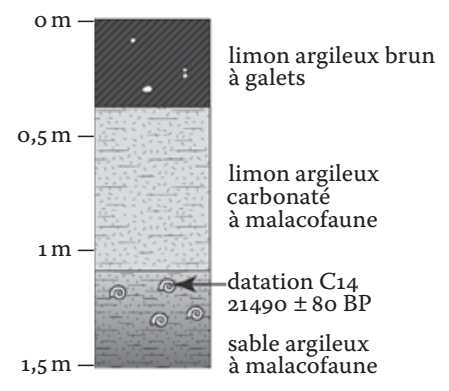
3. Aussonnelle (basse terrasse)

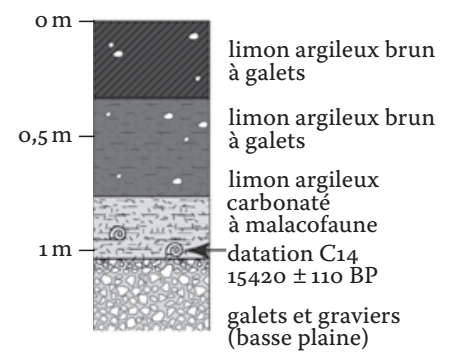

[Fig.2] Carte géomorphologique des terrasses de la Garonne et exemples de logs stratigraphiques relevés dans les couvertures limoneuses et ayant fourni des dates du dernier stade (OIS 2).

[Fig.3] Carte des sites et indices

paléolithiques inférieurs et moyens dans la vallée de la Garonne (points noirs), des indices et sites du Paléolithique supérieur (ronds blancs) et des indices épipaléolithiques et mésolithiques (triangles gris). Extension maximale des glaciers pyrénéens.

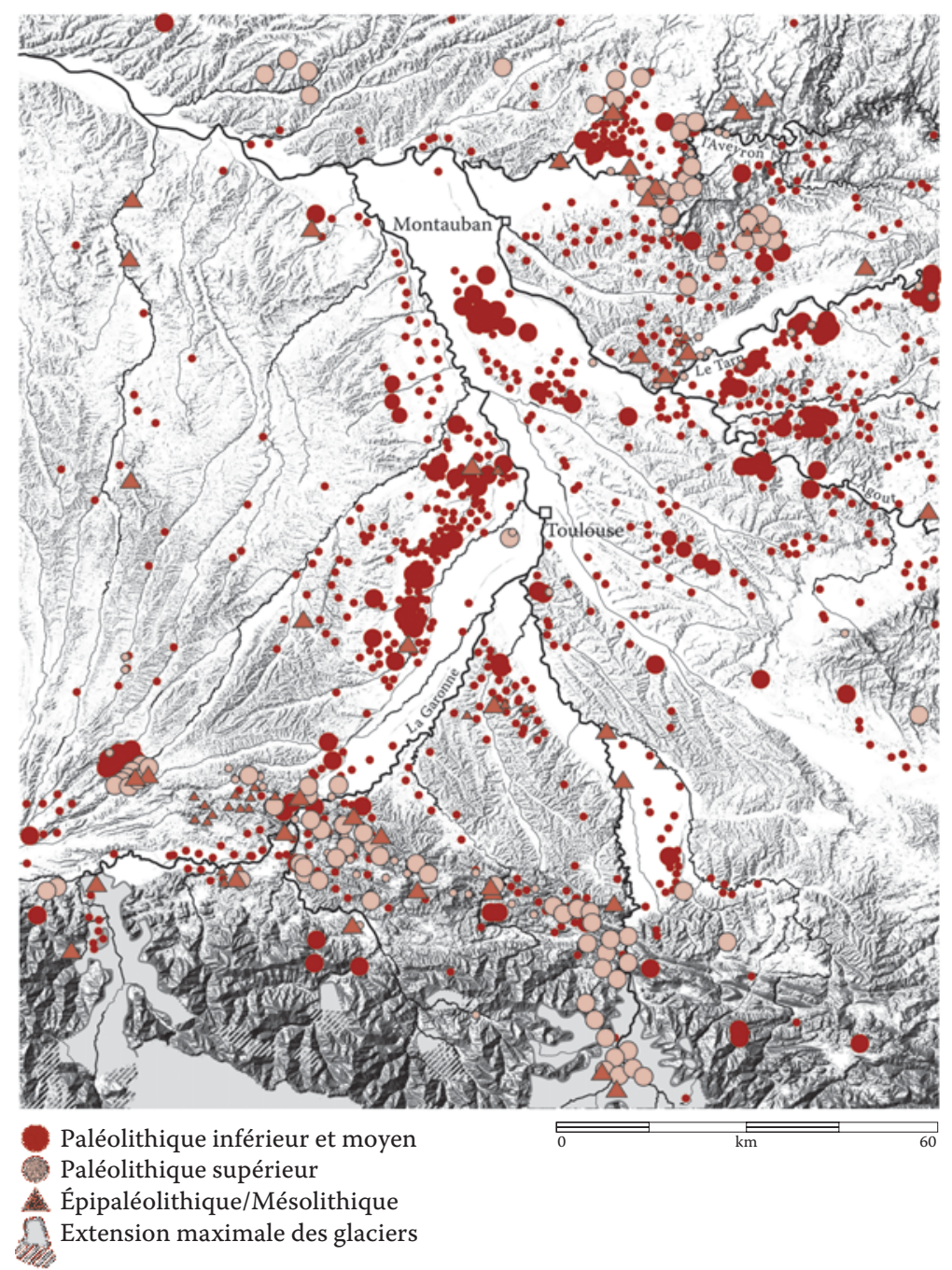


des peuplements durant le reste de la glaciation weischelienne (au Moustérien), mais également pendant les glaciations antérieures. Ici aussi, les implications « culturelles » seront envisagées.

La Garonne dans son cadre géomorphologique.

La vallée de la Garonne est l'élément axial de la structuration du bassin aquitain [Fig.1]. Celui-ci $\left(55000 \mathrm{~km}^{2}\right)$ draine, sur plus de $600 \mathrm{~km}$, la majeure partie du réseau hydrographique du Sud-Ouest de la France. Le puissant fleuve prend sa source dans les Pyrénées, traverse les petites Pyrénées en de profondes cluses, puis entame les molasses tertiaires où le fleuve s'élargit, sa vallée pouvant alors atteindre $25 \mathrm{~km}$ de large au niveau de Toulouse. À partir de cette ville, elle oblique en direction du nord-ouest et rejoint le golfe de Gascogne en contournant, par le nord, le triangle des Landes. Tout le long de son cours jusqu'à l'Atlantique, la Garonne reçoit de nombreux affluents. En rive droite, l'Ariège est alimentée, comme la Garonne, par les Pyrénées. Puis, de l'autre côté du seuil du Lauragais, plusieurs rivières orientées est-ouest descendent des contreforts cristallins du Massif Central et traversent les entablements calcaires au fond de canyons très encaissés. La rive gauche de la Garonne, quant à elle, collecte les eaux des rivières qui parcourent en éventail les coteaux tertiaires de la Gascogne.

La vallée de la Garonne occupe donc une position centrale dans le Sud-Ouest de la France, entre Pyrénées et Massif central. Le domaine molassique aux formes molles est encadré au nord par les plateaux calcaires des Charente, de la Dordogne et du Quercy et, au sud, par les chaînons calcaires qui s'étendent du pays basque jusqu'aux petites Pyrénées. Ces massifs sont entaillés par des canyons encaissés et ils sont largement karstifiés. Ces morphologies ont offert aux populations préhistoriques des ressources diversifiées et une multitude de biotopes aux biocénoses spécifiques au sein desquelles elles ont pu développer et adapter leurs activités saisonnières de subsistance.

L'essentiel du creusement de la vallée de la Garonne s'est opéré au cours du Quaternaire. Les oscillations climatiques sont matérialisées par une alternance de phases de creusement et d'alluvionnement. Ce fonctionnement se traduit, dans le paysage, par un étagement de cinq niveaux principaux de terrasses alluviales, au sein desquelles plusieurs oscillations sont encore perceptibles [Fig.2]. En Midi toulousain, la vallée a un profil dissymétrique très marqué. Les terrasses alluviales forment un large escalier s'étageant sur plus de 200 m d'altitude. Il fait face à la rive droite, souvent abrupte, taillée dans les molasses par le décalage systématique $\mathrm{du}$ fleuve en direction de l'est. Les formations alluviales à galets, épaisses de cinq à six mètres en moyenne, sont recouvertes par des dépôts limoneux dont l'origine a été longtemps discutée. Fluviatiles selon certains, éoliennes pour d'autres, ces formations ont révélé une origine complexe, comme l'avait déjà pressenti Icole (1973), provenant en partie, pour les paliers anciens, du remaniement des formations alluviales altérées (Bruxelles et al.,
2003). La contribution des apports éoliens dans la formation de ces couvertures doit aussi être prise en compte, comme en témoignent les quelques plaquages lœssiques préservés en position d'abri (Hubschman, 1975a et 1975b ; Revel et al., 1978).

Un biais de la recherche? En plus d'importants travaux de prospections archéologiques, le développement de l'archéologie préventive depuis une dizaine d'années a permis d'appréhender le terrain d'une manière radicalement différente. Ces recherches systématiques sont objectives dans le sens où elles répondent à la dynamique d'aménagement du territoire. Ainsi, les interventions, qui couvrent de grandes surfaces ou s'étirent le long de linéaires de plusieurs dizaines de kilomètres, concernent tous les contextes géomorphologiques. En outre, ces opérations sont dotées d'importants moyens logistiques autorisant une étude détaillée du soussol, mais aussi la réalisation de sondages profonds. Ils permettent donc de recouper l'ensemble des terrains susceptibles de contenir des vestiges archéologiques de quelque période que ce soit.

Ainsi, plus de 15 ooo sondages archéologiques ont été effectués depuis une dizaine d'années en Midi toulousain, soit environ 1000 hectares de terrains reconnus. Alors que de très nombreux indices ou sites du Paléolithique inférieur et moyen ont été découverts, complétant une carte archéologique déjà bien fournie, un seul sondage, à quelques kilomètres au sud-ouest de Toulouse, a livré des indices attribuables au Magdalénien (S. Lacombe, communication orale, chantier dirigé par M.-N. Nacfer). Ils confirment par ailleurs la seule découverte ancienne, réalisée à Villeneuve-Tolosane (prospections L. Méroc ; Jarry, 2010 d'après les données de R. Simonnet). Pendant ce temps et à titre de comparaison sur la pertinence méthodologique des recherches, rappelons que le même type de sondage a permis la découverte, dans la région, de sites tardiglaciaires ou postglaciaires, pourtant réputés difficiles à repérer (Amiel, Lelouvier, 2002 ; Lelouvier et al., à paraître). Nous avons même pu trouver, au cœur des terrasses de la Garonne (site de Cassagna 3 à Blagnac, sous la direction de F. Pons), une armature triangulaire mésolithique encore inédite. Ainsi, en dehors de Villeneuve-Tolosane, aucun indice, même isolé, ni même seulement douteux, n'a pu être retrouvé dans une des grandes plaines alluviales du Midi toulousain.

Cette approche permet de répondre, de manière claire, à la première hypothèse : l'absence de Paléolithique supérieur en Midi toulousain ne procède pas d'un biais d'observation ni d'un problème méthodologique.

Un problème de conservation différentielle? Au cours de ces travaux de recherche, nous nous sommes attachés à préciser la nature et l'âge des terrains traversés. Quelques sites et de nombreux indices datant du Paléolithique inférieur et moyen ont été trouvés sur les terrasses de la Garonne, ce qui tend à montrer que tous les vestiges, même anciens, n’ont pas été «purgés» par les phases 


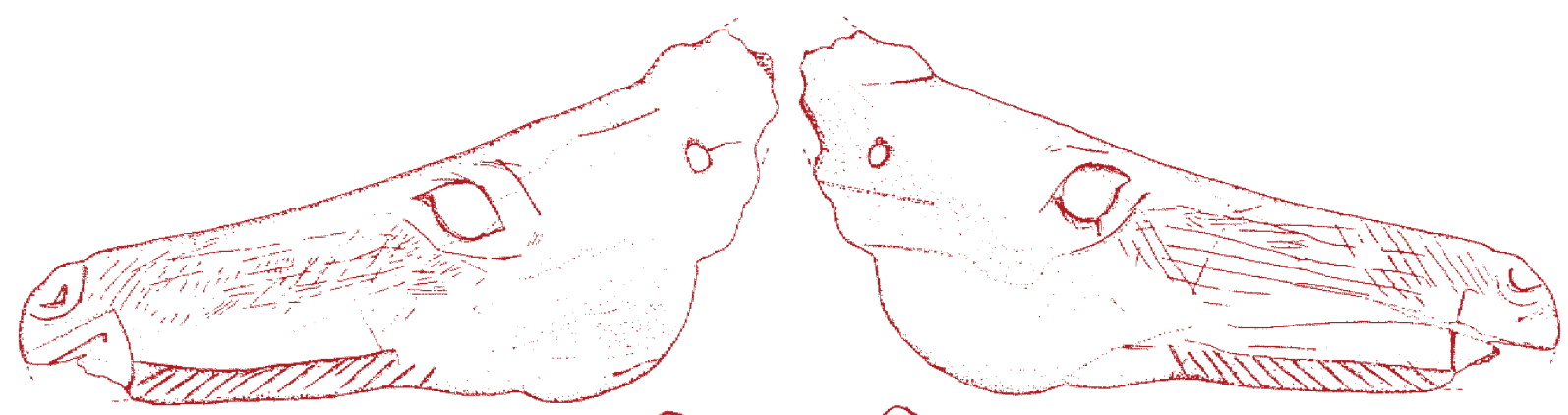

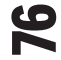
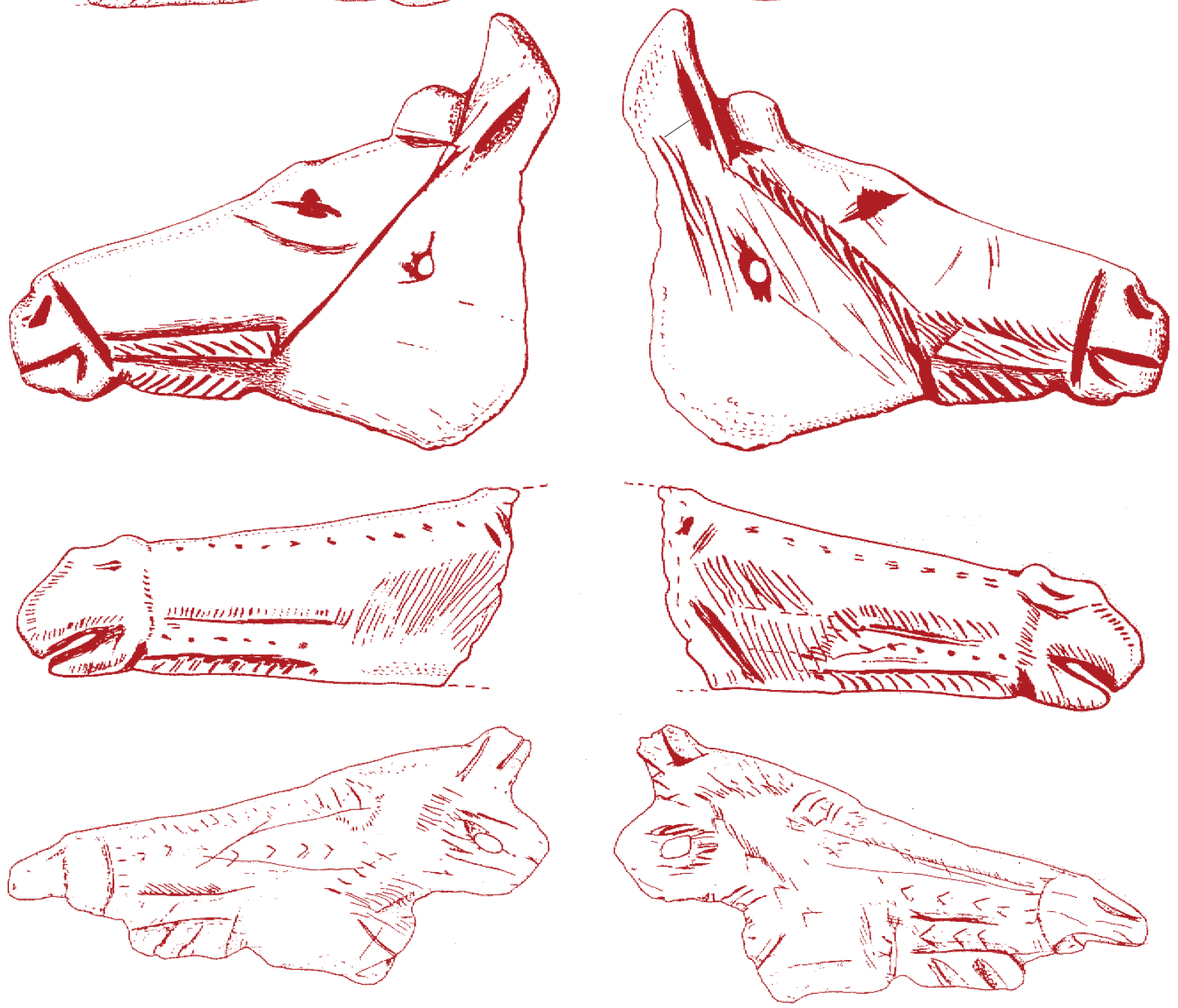

[Fig.4] Contours découpés de têtes de chevaux du Magdalénien moyen pyrénéen. De haut en bas : Isturitz

(Pyrénées-Altlantiques); Enlène

(Ariège); Mas-d'Azil (Ariège) ;

Lortet (Hautes-Pyrénées). 
d'érosion postérieures. Néanmoins, nous avons montré que l'essentiel du matériel paléolithique ne se trouvait pas en position primaire mais qu'il était le plus souvent résidualisé sur le toit de la terrasse graveleuse (Bruxelles et al., 2003). Ce constat traduit donc l'existence de phénomènes d'érosion qui ont en grande partie évacué les anciennes couvertures limoneuses des terrasses.

Les couvertures actuelles ne correspondent donc plus aux limons de débordement propres à chaque terrasse, mais plutôt à des phénomènes de colluvionnement à leur toit. Ces dépôts sont largement enrichis par un saupoudrage de lœss dont on retrouve quelques plaquages et qui sont datés de $20900 \pm 570$ av. notre ère (Hubschman, 1975a et 1975b ; Revel et al., 1978). Ces couvertures sont donc diachroniques des terrasses qu'elles recouvrent. En plusieurs points de la basse plaine et de la basse terrasse, la découverte de malacofaune a fourni un premier calage chronologique de cette formation. Ainsi,les datations ont permis d'estimer la mise en place de ces couvertures au cours de la dernière phase froide du Quaternaire, avec plusieurs résultats : $21490 \pm 85$ av. notre ère (environ 24.150 av. notre ère-environ 23250 av. notre ère) ; $14200 \pm 100 \mathrm{av}$. notre ère (environ $15605 \mathrm{av}$. notre ère-environ 14565 av. notre ère) ; $12505 \pm 50 \mathrm{av}$. notre ère (environ 13011 av. notre ère-12.302 av. notre ère) (Bruxelles et al., 2010 ; Cantournet et al., 2011). Les indications paléo-environnementales déduites de l'étude de ces faunes sont convergentes avec cette attribution chronologique. Elles traduisent un environnement froid de type steppique.

Ces observations, largement étayées sur la basse plaine et la basse terrasse de la Garonne, montrent donc que les terrains de la fin du dernier stade (OIS 2 ) susceptibles de contenir des vestiges du Paléolithique supérieur sont largement présents en Midi toulousain. Ce sont d'ailleurs ces terrains qui constituent l'essentiel des couvertures limoneuses des terrasses de la Garonne. Les sédiments et la malacofaune qu'ils contiennent, et a fortiori les vestiges paléolithiques, n’ont donc pas été évacués par l'érosion. Il est donc possible, comme pour la question du déficit en recherches, d'écarter le biais taphonomique comme explication de l'absence de vestiges du Paléolithique supérieur.

Un choix d'implantation! Si ce n'est ni un biais de la recherche, ni un biais taphonomique, les cartographies critiques des sites et indices que nous allons commenter ci-après sont donc des représentations assez fidèles statistiquement, des peuplements paléolithiques de cette portion du Sud-Ouest de la France.

Ainsi, il existe un contraste frappant entre la carte de répartition des sites et indices attribuables au Paléolithique inférieur et moyen et celle qui recense les vestiges du Paléolithique supérieur [Fig.3]. Pour cette dernière période, les sites sont tous, ou presque, en dehors des grands couloirs alluviaux de ce secteur. Seuls quelques rares indices aurignaciens sont signalés dans les vallées du Tarn et l'Aveyron (Alaux, 1970) ou au débouché de la Cluze de Boussens (Méroc, 1963 ; Clottes, 1976 ; Jarry, 2010). L'unique occurrence au centre du dispositif reste celle de Villeneuve-Tolosane.Il faut ensuite remonter sur les coteaux molassiques pour rencontrer des occupations du Paléolithique supérieur, comme à l'interfluve Garonne-Ariège, où des prospections régulières ont permis de repérer des gisements où les sites magdaléniens dominent par la qualité et la quantité (prospections, R. Scanduzzi) ou au nord, dans les Serres de l'Agenais (Le Brun-Ricalens, 1988). Le reste, c'est à dire l'immense majorité, est concentré dans les zones karstiques aux profonds canyons du piémont pyrénéen et des causses).

Quoi qu'il en soit, en plaine, on ne compte encore aujourd'hui absolument aucun indice rapportable au dernier optimum glaciaire (épi-Gravettien, Solutréen, Badegoulien). Pour comparaison, la carte des sites postglaciaires indique une répartition différente. Effectivement, tout en comptant beaucoup moins de données, elle correspond clairement à une toute autre logique, avec une reconquête des espaces, dont l'amorce commence dès le Tardiglaciaire. La forêt tempérée gagne alors sur la steppe froide.

Il nous reste à nous rendre à l'évidence, les groupes du Paléolithique supérieur n'auraient donc pas ou très peu fréquenté la moyenne vallée de la Garonne, ni même les basses vallées de l'Ariège, du Tarn et de l'Aveyron. Et nous pouvons reprendre ici l'observation de Jacques Jaubert pour sa troisième proposition de réponse : « un réel manque d'établissements de plein air opposerait de manière radicale des plaines ou des coteaux désertiques aux plateaux et chaînons calcaires recelant les innombrables gisements sous abri, tant au nord (...) qu'au sud » (Jaubert, 2002, p. 50). Il anticipait, sans pouvoir cependant alors prendre position, sur ce que nous pouvons désormais poser comme une réalité. Nous avons pu démontrer qu'il y avait bien un hiatus de peuplement dans la vallée de la Garonne pendant le Paléolithique supérieur, soit pendant tout le Dernier Maximum Glaciaire. Cependant, les indices que nous avons relevés pourraient correspondre à des incursions pendant les phases interstadiaires (par exemple Dansgaard/ Oeschger 1 pour le Magdalénien, DO 3 à 7 pour l'Aurignacien). Ce schéma est similaire à celui observé dans les contrées septentrionales de l'Europe qui ont été abandonnées pendant les phases froides et sèches (Roebroeks et al., 1992 ; Antoine et al., 2003).

\section{Le Dernier Maximum Glaciaire dans la vallée} de la Garonne. La concordance entre le Dernier Maximum Glaciaire et l'abandon de la vallée de la Garonne par les populations peut donc logiquement être interprétée par une relation de cause à effet. Le Dernier Glaciaire est marqué par des variations climatiques brusques et une baisse sensible des températures (Dansgaard et al., 1993) accompagné d'un volume important des glaces polaires (Peltier, 1994). Les impacts environnementaux notables pour l'Europe sont, outre les températures basses, une baisse du niveau marin, une modification des courants océaniques, des courants atmosphériques plus turbulents, une banquise d'hiver qui atteint le golfe de Gascogne, un vaste 


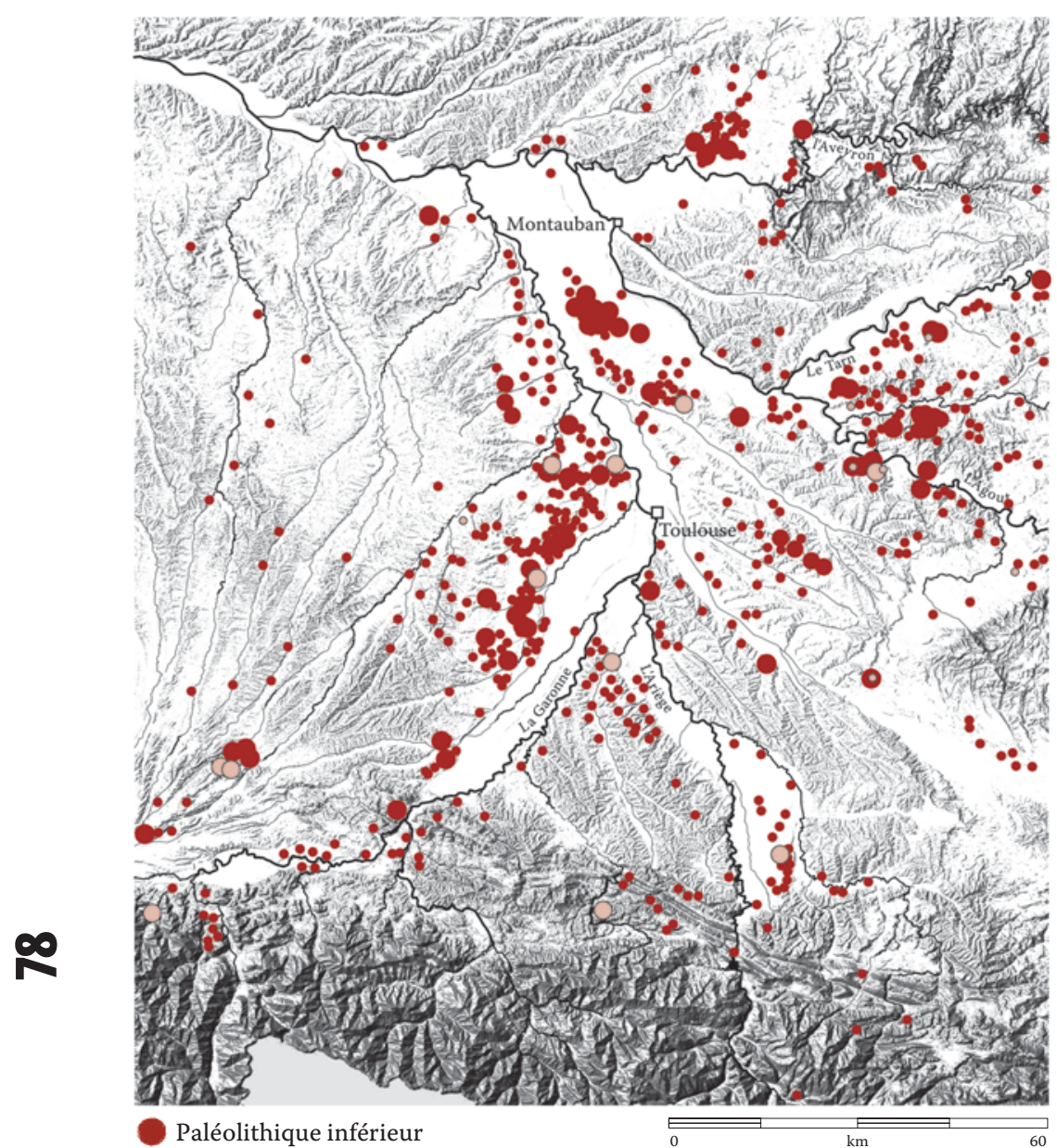

Paléolithique moyen ancien

[Fig.5] Carte des sites et indices

Paléolithique inférieur (ronds noirs)

et du Paléolithique moyen ancien

(ronds blancs).

[Fig.6] Carte des sites et indices du

Paléolithique moyen (ronds blancs) avec les sites à bifaces et apparentés isolés (ronds noirs).

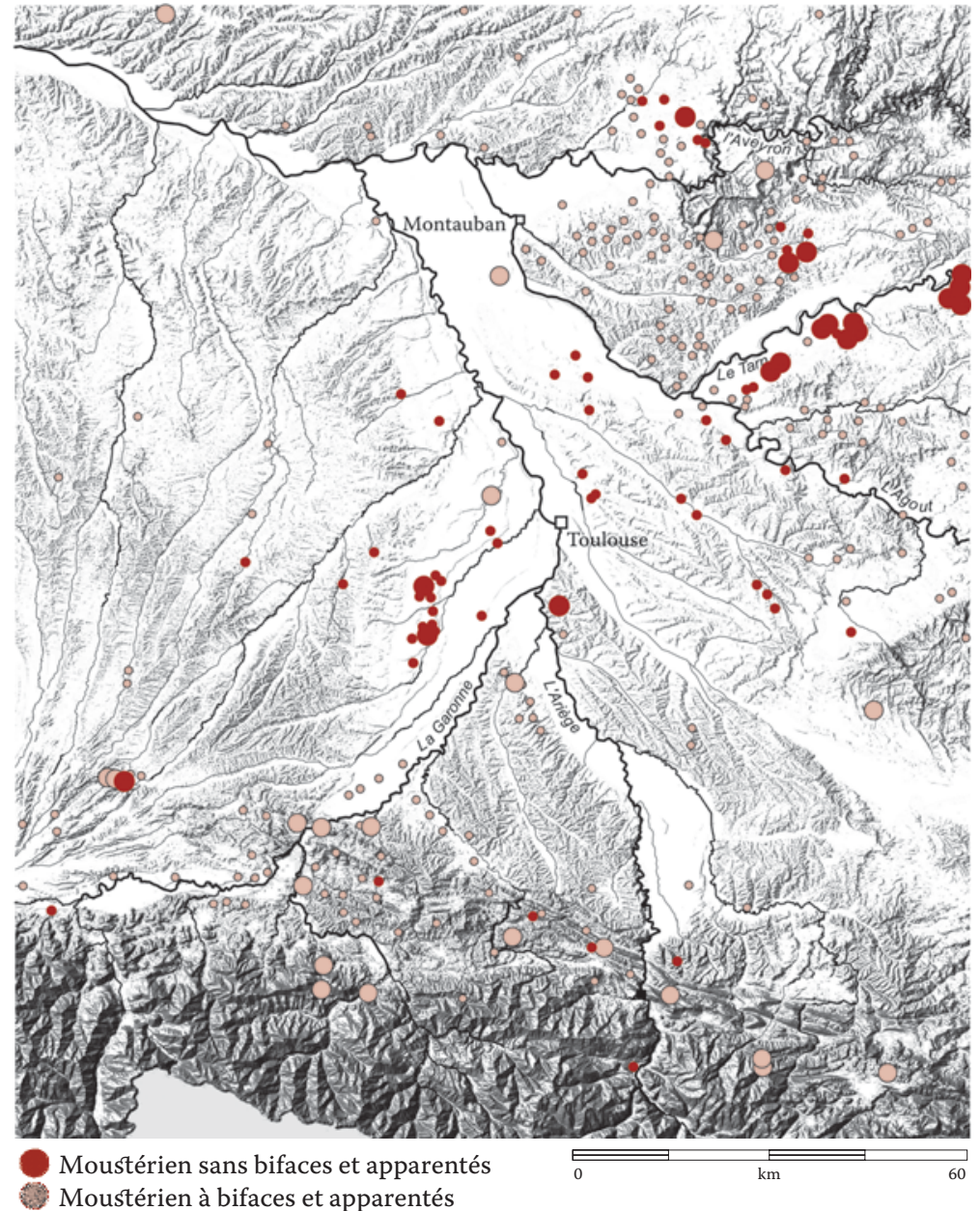


désert polaire et un pergélisol très méridional. L'Aquitaine est alors encadrée par des glaciers plus ou moins imposants sur les Pyrénées, le Massif Central et l'Aubrac. Les langues glaciaires peuvent descendre assez bas dans les vallées. Les paysages et les biocénoses, en mosaïque, sont profondément modifiés pour les contrées nordiques de l'Europe, mais aussi plus méridionales.

La biomasse animale est décrite comme étant très faible, très mosaïquée, notamment pendant le Pléniglaciaire, qui connaît même une réduction de la stature des animaux (Delpech, 1988, 1999). On sait par ailleurs depuis longtemps que les Landes de Gascogne étaient, pendant les périodes froides et arides du Dernier Glaciaire, un vaste désert sableux (Klingebiel, Legigan, 1985 ; Bertran et al., 2009 ; Bertran et al., à paraître). L'erg landais connaît des phases de dunes actives et des vents puissants provoquant d'intenses déflations de direction globalement ouest-est (axes des dunes paraboliques). Cette zone inhospitalière ne crée cependant pas une frontière incontournable pour les groupes du Paléolithique, puisqu'elle est cantonnée à l'ouest du bassin aquitain, dans un vaste triangle adossé au golfe de Gascogne dont les rivages étaient bien plus éloignés lors des phases froides. Ce désert constituerait, au pire, une zone à contourner pour relier les piémonts pyrénéens et les contreforts calcaires du Massif Central.

De même, et l'absence de vestiges du Paléolithique supérieur en démontre la rigueur, le couloir garonnais devait, en phases froides, connaître des conditions peu favorables à une implantation pérenne des groupes humains. Le paysage ouvert steppique et l'importance des vents, accentués par l'effet venturi du couloir alluvial (les lœss sont présents dans toute la vallée, jusqu'au seuil du Lauragais) devaient rendre cette zone particulièrement inhospitalière. Le couloir garonnais, prolongeant le désert landais, est alors une zone non occupée, ou très peu fréquentée, en faveur des zones d'abris et des refuges karstiques des piémonts montagnards. Dans ce cas, le terme de « refuge » semble adapté, à l'inverse des grandes plaines septentrionales où, lorsque les conditions environnementales se sont dégradées, il n'y avait pas de zones de repli à proximité. Il fallait donc s'adapter ou éventuellement migrer sur de bien plus grandes distances.

La vallée de la Garonne devait ainsi constituer une zone de « frontière », séparant les groupes humains en deux ensembles distincts, repliés, dans les piémonts plus protégés et donc plus accueillants des Pyrénées et du Massif Central. Cet isolement est sensible entre les deux rives à travers les formes culturelles endémiques, dont certaines ont été observées depuis déjà longtemps. Nous citerons ici quelques exemples car il faudra nécessairement pousser plus loin l'analyse. Le Gravettien pyrénéen connaît une forte originalité des groupes de l'ouest et du centre des Pyrénées (Foucher, 2004; Foucher et al., 2008). L'Est, par contre, aurait des affinités différentes, stigmatisées par la présence de fléchettes, comme en Charente et Dordogne (Pessese, 2008, fig. 1 ; Foucher et al., 2008). De même, la phase rayssienne du Gravettien reste contingentée au nord de la Garonne (Klaric, 2008), la Garonne marquant une des frontières de la mosaïque culturelle du gravettien (Klaric et al., 2009). Le Solutréen pyrénéen, avec son «style particulier » est marqué, notamment, par les pointes asymétriques, dites de Montaut; les pointes à bases concaves, certaines pointes à cran et pédoncule court et courbe, dégagé par une simple encoche ou un type de feuille de saule à base convexe, rectiligne ou transversale (Cartailhac, 1896 ; Begouën, 1935 ; Foucher, San Juan, 2002 ; Foucher, 2004). Dans l'art magdalénien, des «marqueurs ethniques » sont décrits entre Pyrénées, Cantabres et Dordogne-Charente (Leroi-Gourhan, 1981). Ainsi, dans le monde pyrénéen (Clottes, 1976, 1989 et 1996), les baguettes demi-rondes de type «d'Isturitz», ne sont présentes que dans la partie occidentale et centrale des Pyrénées, de même que les contours découpés [Fig.4], qui existent également en Espagne, mais aussi les propulseurs sculptés du type propulseur au faon du Mas d'Azil. Nous pouvons en outre ajouter la gravure sur argile et le modelage, tout comme les contours noirs, la bichromie ou l'association gravure/peinture pour l'art pariétal. Il avait même été remarqué des sousrégions au sein de ce Magdalénien pyrénéen (Sacchi, 1986; Vialou, 1986). Ces originalités ont cependant été reprises plus récemment, unifiant Cantabres et Pyrénées dans un même ensemble (Fritz, Tosello, 2005, fig. 12). À la fin du Magdalénien, il a été remarqué que les représentations féminines schématiques type Lalinde-Gönnersdorf (symbole pourtant de l'unité européenne de cette culture), présentent cependant, au nord de la Garonne, une nuance spécifique avec le dessin d'un sein (Sentis, 2005). À l'Aurignacien, si des pistes sont à rechercher avec les ornements personnels (Vanhaeren, d'Errico, 2006), les liens ne devaient pas être assez distendus pour faire apparaître des particularismes prononcés (Bon et al., 2005 ; Bordes et al., 2005). En effet, il a été noté, par l'étude de l'origine et de la gestion des silex allochtones, des liens étroits entre les différents territoires aquitains. Rappelons, à ce sujet, et c'est pour cela que nous préférons parler de «frontière » plutôt que de «barrières », que, durant tout le Paléolithique supérieur, les liens ne sont pas rompus entre les deux rives. En témoignent la synchronie des grands courants culturels, mais aussi les circulations des silex pour lesquelles, quel que soit le mode d'approvisionnement envisagé (acquisition directe, échanges), la distance est insuffisante pour séparer complètement les deux rives (Simonnet, 1996, 1998 et 2007 ; Foucher, 2004 ; Le BrunRicalens et al., 2004 ; Foucher, San Juan, 2005 ; Séronie-Vivien et al., 2006 ; Bourguignon, Turq, 2008 ; Foucher et al.,2008 ; Séronie-Vivien, 2009).

Et avant? Pour l'instant, nous avons surtout évoqué le Paléolithique supérieur car les vestiges archéologiques relevant de cette période sont faciles à identifier et la résolution chronologique nécessaire est suffisante. Si nous partons de ce point, c'est à dire du constat d'une très faible, voire d'une absence d'implantation de groupes du Paléolithique supérieur dans la vallée de la Garonne, dont 

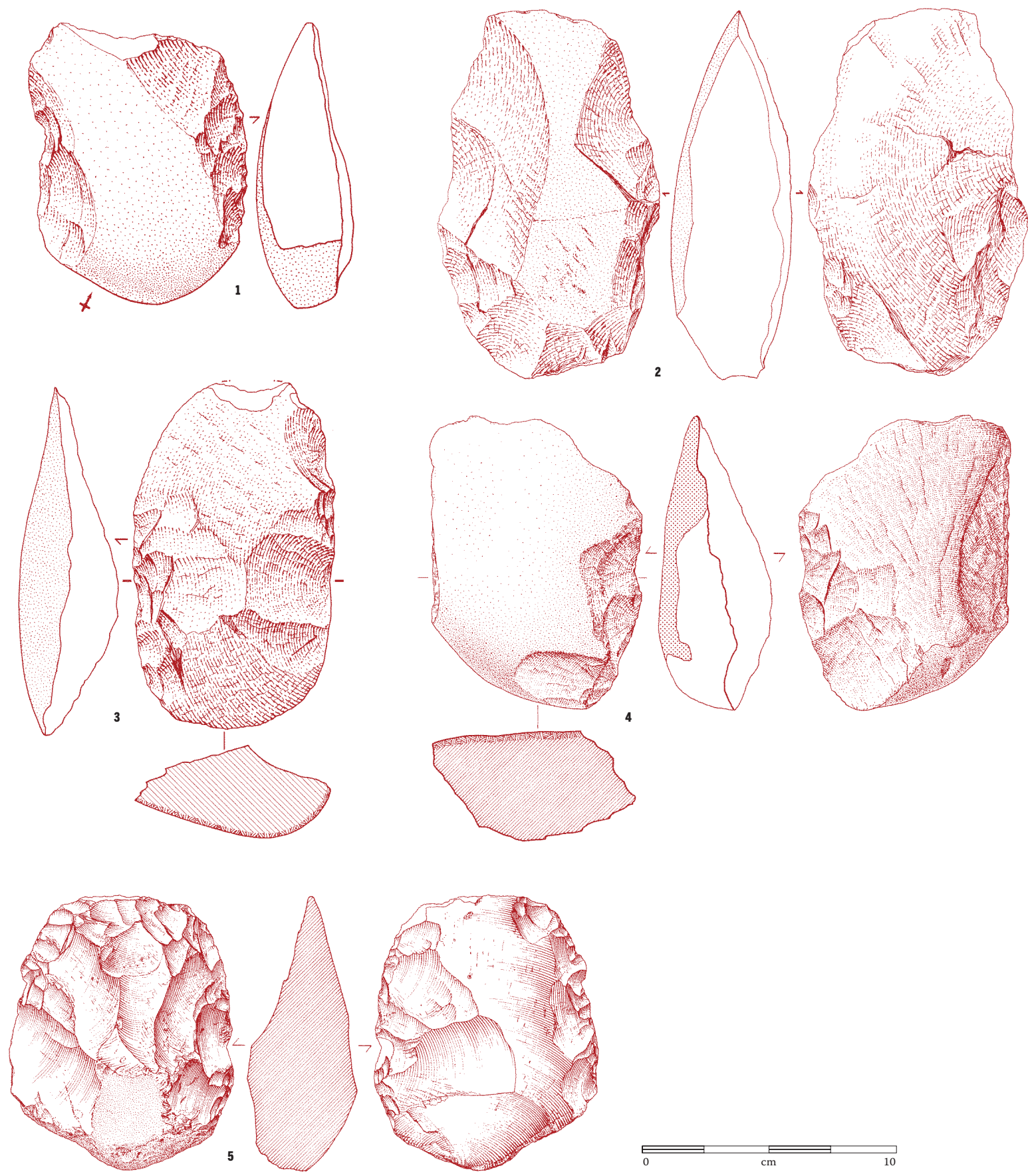

[Fig.7] Hachereaux ; 1 : quartzite,

Acheuléen moyen « archaïque "

de La Rominguière (Cornebarrieu,

Haute-Garonne) ; 2 : quartzite,

Acheuléen moyen du niveau inférieur

de Pentens (Martres-Tolosane,

Haute-Garonne); 3 : Acheuléen

moyen de la série $B$ de Bichou

(Montaigut-sur-Save, Haute-

Garonne); 4 et 5 : silex et quartzite,

Vasconien du Turon de Calavanté

(Hautes-Pyrénées). 
le déterminisme environnemental serait la cause, il est tout à fait envisageable que le même modèle puisse être avancé pour les autres péjorations climatiques de la glaciation weischelienne. Nous avons donc essayé d'affiner les cartes de répartitions des sites et indices pour le Paléolithique inférieur et moyen [Fig.5]. Celle du Paléolithique inférieur montre encore une occupation très intense de la vallée de la Garonne et de ses marges. Au Paléolithique moyen ancien (Acheuléen supérieur), les quelques références isolables suivent le même schéma. Nous avons ensuite séparé les sites moustériens à bifaces de ceux n'en contenant pas [Fig.6]. Dans le second cas, un seul site avéré est localisé dans la vallée. C'est celui de Bel Soleil, à l'ouest de Toulouse, et il est rapportable au début du stade 3 (Bruxelles et al., 2008 ; Jarry, 2010). Le reste, comprenant le Moustérien récent, a une distribution des sites et indices similaire à celle observée au Paléolithique supérieur. Ainsi, et malgré quelques distorsions qui pourraient être provoquées par la difficulté bien réelle qu'il peut y avoir à «classer» une industrie en «Moustérien récent », l'image qui en ressort est sans équivoque celle d'un abandon des habitats permanents de la plaine, avec une incursion dans une phase de réchauffement qui succède au froid du stade isotopique 4.

La carte de distribution des industries moustériennes à bifaces et apparentés est plus complexe. Les sites avérés sont rares et mal ou non datés. Ils semblent répartis plutôt aléatoirement au sein du territoire concerné. Cependant, en affinant l'analyse, il peut être isolé un premier groupe de sites typiquement moustériens de tradition acheuléenne dans la vallée du Tarn (Tavoso, 1986). Cet auteur ne voit pas de raison géomorphologique ou de disponibilité des matières premières dans les hautes vallées pour expliquer cette présence du MTA. À l'opposé, peu de sites pyrénéens sont signalés, parmi lesquels on peut noter l'industrie de la couche $3 \mathrm{z}$ de Coupe-Gorge à Montmaurin (Gaillard, 1979), datée par sa faune chaude de la fin du Dernier Interglaciaire et du début du Dernier Glaciaire (OIS 5 ). Il faut aller plus à l'ouest pour retrouver des industries vasconiennes du stade 5 (Olha 1 et 2), puis toute la série des sites du Vasconien récent pyrénéen (OIS 3 ; Mourre et al., 2008 ; Deschamps, 2008). Quant à elle, la vallée de la Garonne compte quelques exemples d'indices et de sites. Ces derniers, dont l'homogénéité reste cependant douteuse, comprennent quelques hachereaux et pourraient être rattachés au Vasconien (La Ferratière : Simonnet, inédit ; La Tourrasse ; Pinos, 1999).

Un premier scénario peut être proposé : les occupations du MTA « classique» du Tarn pourraient correspondre, comme pour celles du Périgord et de la Charente (Soressi, 2002 ; Soressi et al., 2008), aux occupations « refuges » des stades 4 et surtout 3. Le Vasconien tardif pyrénéen serait alors son symétrique, mais issu d'un substrat culturel différent. En effet, à l'interglaciaire eémien, les industries à bifaces et apparentés se rencontrent dans tout l'ouest européen, montrant des formes de continuité avec les substrats locaux (septentrional, micoquien, méridional, ibérique). Ainsi, les occupations moustériennes à bifaces de la plaine Garonnaise pourraient être rapportables à l'interglaciaire eémien ou un interstade du stade 5 (le stade isotopique 3 ne peut pas cependant être exclu). Les occupations en zones de refuges de piémont seraient, si on se réfère au schéma décrit pour le Paléolithique supérieur, associables aux phases froides.

Partant du postulat selon lequel, pendant la glaciation weischelienne, en dehors de quelques interstadiaires, la vallée de la Garonne n'était pas favorable aux occupations humaines, il est rationnel de se poser la question pour les glaciations antérieures. En effet, par analogie, on peut supposer le même abandon pour les stades isotopiques $6,7.4,8,10 \ldots$

Même si le détail des glaciations antérieures n'est pas identique à celui du Dernier Glaciaire, il a pu être observé des désertifications régulières du Nord de l'Europe, avec même peut-être une accentuation pour le stade isotopique 6 (Gamble, 1986 ; Roebroeks et al., 1992 ; Antoine et al., 2003). Certes, ce stade est un peu moins froid que le Dernier Glaciaire, mais il est plus long et moins entrecoupé de phases plus tempérées (Coleoni, 2009). Ainsi, à titre d'hypothèse, il peut être supposé que les nombreux témoins d'occupations acheuléennes relèvent des phases clémentes alors que les occupants des deux rives sont «en contact».

Si l'on poursuit ce raisonnement, et partant du fait que les groupes du Paléolithique inférieur et moyen ancien sont réputés bien moins mobiles que ceux du Paléolithique moyen récent et encore moins que ceux du Paléolithique supérieur, il est possible d'imaginer que le corridor garonnais pouvait constituer une frontière culturelle pour ces groupes lors des phases froides. Or, à l'Acheuléen, la Garonne constitue la limite nord de l'Acheuléen ibérique (Turq et al., 2010), lui-même ayant des affinités avec l'Acheuléen maghrébin. Celui-ci est marqué, notamment, par la présence des hachereaux [Fig.7]. On notera aussi, comme différence, la présence du débitage trifacial au nord de la frontière garonnaise (Boëda, 1991), que nous ne connaissons pas à ce jour au pied des Pyrénées. Nous ne discuterons pas ici du caractère déterminant ou non des matières premières pour ce type d'outillage sur grands éclats, mais toujours est-il que la séparation régulière des groupes humains lors des pulsations climatiques n'a pu qu'accentuer des caractères propres à chaque rive. Les hachereaux pourraient être alors l'une des marques la plus visibles de cet endémisme, avec un Acheuléen ibérique qui remonterait jusqu'à la vallée de la Garonne. Nous avons vu qu'il pourrait y avoir ici, dans ce substrat ibérique, une origine de la différenciation et de l'accentuation des caractères du Vasconien par rapport au MTA lui-même en position isolée sur l'autre rive.

Vers une nouvelle perception des territoires... La question du rôle de la vallée de la Garonne n'a jamais véritablement été posée en l'absence de moyens d'investigation ou de l'ingratitude des terrains. Pourtant, les découvertes archéologiques sont nombreuses et de grands hiatus ont déjà été observés, notamment pour le Paléolithique supérieur. Comme

.


nous l'avons vu plus haut, cette absence ne procède pas d'un biais d'observation ni d'une conservation différentielle des terrains imputables à l'histoire géomorphologique. Nous avons pu montrer aussi que l'absence presque totale d'occupations du Paléolithique supérieur et du Paléolithique moyen récent, en dehors de rares éléments correspondant à des améliorations climatiques, découle d'un abandon de la vallée de la Garonne à ces époques. Nous en déduisons que les facteurs climatiques, très rudes pendant les phases glaciaires, devaient rendre particulièrement inhospitalier ce large couloir, repoussant alors les populations préhistoriques dans les secteurs plus protégés qui encadrent la vallée.

Les implications qui découlent de ces conclusions sont multiples. Ainsi, le Sud-Ouest ne constituerait pas, dans son ensemble, un «refuge» pour les populations septentrionales chassées par le désert glaciaire. Les péjorations climatiques s'y ressentent aussi très fortement, mais de manière différentielle selon le contexte topographique, ce qui a manifestement guidé les choix d'implantation. Il conviendrait donc de reconsidérer les modalités d'occupation du vaste territoire aquitain, avec des populations «contraintes » dans des vallées encaissées, même si, nous l'avons vu, des contacts sont possibles et «déployées » dans les plaines en conditions interglaciaires.

Enfin, il est légitime d'imaginer que cette adaptation aux conditions environnementales s'est répétée au gré des variations climatiques quaternaires. Il n'y a pas de raison que l'influence climatique forte constatée pour le Paléolithique supérieur n'ait pas eu les mêmes conséquences dans les phases plus anciennes. Ainsi, l'histoire du peuplement préhistorique du Sud-Ouest se serait donc réalisée au gré de pulsations où alternent la contraction des espaces occupés pendant les phases glaciaires et leur dilatation pendant les interstades. La vallée de la Garonne constituerait, alternativement, un pivot favorisant la circulation et une frontière limitant les contacts nord/sud, en fonction des conditions environnementales qui se sont succédées au cours des fluctuations climatiques du Pléistocène. Le rôle de «frontière» joué par le couloir garonnais pourrait être la cause de certaines nuances «culturelles» endémiques, causées par des positions d'isolements connues de part et d'autre de la Garonne et ce, pendant tout le Paléolithique.

AlAUX, J.-J., 1970 : « Nouvelles stations aurignaciennes de surface du Tarn ", Travaux et Recherches Fédération tarnaise de spéléoarchéologie 7, P. 10-17.

AMiel C., Lelouvier L.-A. (éds.), 2002 : Gisements postglaciaires en bas Quercy. Variabilité des espaces et des statuts de deux occupations mésolithiques sauveterriennes de plein air, Archives d'Écologie Préhistorique, Centre d'Anthropologie, Inrap, Toulouse, 205 p.

Andrieu V., Hubschman J., Jalut G., Herail G., 1988 : « Chronologie de la déglaciation des Pyrénées françaises ", Bulletin de l'Association Française pour l'étude du Quaternaire, 2-3, p. 55-67.

Antoine P., Auguste P., Bahain J.-J., Coudret P., Depaepe P., Fagnart J.-P., Falguère C., Fontugne M., Frechen M., Hatte C., LAMOtTe A., Laurent M., Limondin-Lozouet N., Locht J.-L., Mercier N., Moigne A.-M., Munaut A.-V., Ponel P., RousseAu D.-D., 2003 : Paléoenvironnements pléistocènes et peuplements paléolithiques dans le bassin de la Somme (nord de la France), Bulletin de la Société Préhistorique Française, 100/1, p. 5-28.

Cantournet, C., Jarry M., Pallier C., Pancin S., 2011 : Chemin des Palanques (Bessens, 31), Rapport de Diagnostic Archéologique, Inrap, Paris.
Banks W.E., D'errico F., Dibble H.L., Krishtalka L., West D., Olszewski D.I., Peterson A.T., Anderson D.G., Gillam J.C., Montet-White A., Crucifox M., Marean C.W., SAnChez-Goni M.-F., WOLFARTH B., VANHAEREN M., 2006 : « Eco-cultural niche modeling: new tools for reconstructing the geography and ecology of past human populations ", PaleoAnthropology, 4, p. 68-83.

Banks W.E., D'errico F., Peterson A.T., Vanhaeren M., Kageyama M., Sepulchre P., Ramstein G., Jost A., Lunt D., 2008 : « Human ecological niches and ranges during the LGM in Europe derived from an application of eco-cultural modelins », Journal of Archaeological Science, 35, p. 481-491.

BanKs W.E., Zilhão J., D'errico F., Kageyama M., Sima A., RONCHITELLI A., 2009: « Investigating links between ecology and bifacial tool types in Western Europe during the Last Glacial Maximum », Journal of Archaeological Science, 36, p. 2853-2867.

BÉGouËN H., 1935 : « Le Solutréen dans les Pyrénées », Revue Anthropologique, 4-6, p. 126-136.

Bertran P., Allenet G., Gé T., Naughton F., Poirier P., SanchezGoNI M.-F., 2009: « Coversands and pleistocene palaeosols in the Landes region, southwestern France ", Journal of Quaternary Science, 24 (3), p. 259-269.

Bertran P., Banks W.E., Baterman M.D., Demars P.-Y., Langlais M. LENOIR M., à paraître : « Les Landes de Gascogne : désert périglaciaire et frontière culturelle au Paléolithique » in Des Climats et des Hommes: Glaciologie, climatologie, archéologie, histoire, Actes du colloque de Paris, 19-21 nov. 20o9, Paris, Inrap, Météo-France, Cité de la Science (éds.).

Bocquet-Appel J.-P., Demars P.-Y., 20ooa : « Population kinetics in the upper palaeolithic in western Europe », Journal of Archaeological Science 27, p. 551-570.

BOCQUET-APPEL J.-P., DEMARS P.-Y., 200ob : « Neanderthal contraction and modern human colonization of Europe », Antiquity, 74/285, p. 544-552.

Bocquet-Appel J.-P., Demars P.-Y., NoIret L., Dobrowsky D., 2005 "Estimates of Upper Palaeolithic meta-population size in Europe from archaeological data ", Journal of Archaeological Science, 32, p. 1656-1668

Bö̈DA E., 1991 : « Approche de la variabilité des systèmes de production lithique du Paléolithique inférieur et moyen: chronique d'une variabilité attendue », Technique et Culture, 17-18, p. 37-79.

Bon F., Simonnet R., Vézian J., 2005 : «L'équipement lithique des aurignaciens à la Tuto de Camalhot (Saint-Jean-de-Verges, Ariège). Sa relation avec la mobilité des groupes et la répartition de leurs activités dans un territoire ", in JAUBERT J., BARBAZA M. (ÉD.), Territoires, déplacements, mobilités, échanges durant la Préhistoire. Terres et hommes du Sud, Paris, CTHS, p. 173-184

Bordes J.-G., Bon F., Le Brun-RiCAlens F., 2005 : « Le transport des matières premières lithiques à l'Aurignacien entre le nord et le sud de l'Aquitaine: faits attendus, faits nouveaux », in JAUBERT, J., BARBAZA, M. (ÉDS.), Territoires, déplacements, mobilités, échanges durant la Préhistoire. Terres et hommes du Sud, Paris, CTHS, p. 185-198.

BourguignON L., TurQ A., 2008 : « Territoires lithologiques et axes de circulation au Paléolithique dans le Nord-Est aquitain ", Archéopages, 21, p. 8-9.

BrUXelles L., JARRY M., 2011 : « Climatic conditions, settlement patterns and cultures in the Paleolithic: The example of the Garonne Valley (southwest France) », Journal of Human Evolution, 61, p. 538-548.

Bruxelles L., Berthet A.-L., Chalard P., Colonge D., Delfou G., Jarry M., Lelouvier L.-A., Arnoux T., Onezime O., 2003 : «Le Paléolithique ancien et moyen en Midi toulousain : nouvelles données et perspectives de l'archéologie préventive», Paléo, 15, p. 7-28.

Bruxelles L., JARRY M., Bevilacqua R., 2008 : « Un nouveau jalon pour l'approche contextuelle du Paléolithique moyen en Midi toulousain : Bel-Soleil à Cornebarrieu (Haute-Garonne, France) », in Jaubert J., Bordes J.-G., Ortega I. (ÉDs.), Les Sociétés $d u$ Paléolithique dans le Grand-Sud-Ouest de la France : nouveaux gisements, nouveaux résultats, nouvelles méthodes, Journées SPF, Université de Bordeaux 1, Talence, 24-25 nov. 2006, Société Préhistorique Française (mémoires, XLVII), p. 174-188.

Bruxelles L., Pons F., Magnin F., Bertrand A., 2010 : « Âges et modalité de la mise en place de la couverture limoneuse de la basse plaine de la Garonne d'après l'exemple du site de Fontréal (Castelnaud'Estrétefonds, Haute Garonne) », Quaternaire, 21, p. 339-348.

BRUXELLES L., JARRY M., 2011 : « Climatic conditions, settlement patterns and cultures in the Paleolithic: The example of the Garonne Valley (southwest France) », Journal of Human Evolution, 61, 538-548.

Buisson D., Fritz C., Kandel D., Pincon G., Sauvet G., Tosello G. 1996 : « Les contours découpés de têtes de chevaux et leur contribution à la connaissance du Magdalénien moyen ", Antiquités Nationales, 28, p. 99-128.

Calvet M., 2004: « The Quaternary glaciation of the Pyrenees », in EHLERS J., GibBARD Pl. (ÉDS.), Quaternary Glaciations-Extent and Chronology, part I : Europe, Elsevier, p. 119-128.

Cartailhac E., 1896 : «Quelques faits nouveaux du Préhistorique ancien des Pyrénées », L'Anthropologie, 7, p. 309-318.

Clottes J., 1976 : « Les civilisations du Paléolithique supérieur dan les Pyrénées », in DE LuMLey H. (ÉD.), La Préhistoire Française, I,1, Paris, CNRS éd., p. 1214-1231.

Clottes J., 1989 : « Magdalénien des Pyrénées », in Rigaud J.-Ph., Le Magdalénien en Europe. La structuration du Magdalénien, Actes du colloque de Mayence, 1987, XI congrès U.I.S.P.P., Liège, ERAUL, 38, p. 281-357. 
Clottes J., 1996 : « Le Magdalénien des Pyrénées, un groupe culturel homogène ", in Thiault N.H., Roy J.B. (DIR.), L’Art préhistorique des Pyrénées, Catalogue d'exposition, Musée des Antiquités Nationales, avril-juillet 1996, M.A.N./ éd. Réunion des Musées Nationaux, p. 36-59.

Coleoni F., 2009: On the Late Saalian glaciation (160-14o ka): a climate modeling study, Ph.D. dissertation, Université de Grenoble et Université of Stockholm, 59 et 147 p.

CONARD N. J., 1992: Tönchesberg and its position in the Paleolithic prehistory of northern Europe. Römisch-Germanisches Zentral Museum, Forschungsinstitut für vor- und Frühgeschichte, Monographien, Band 2o, $176 \mathrm{p}$.

Dansgaard W., Jonhnsen J.S., Clausen H.B., Dahl-Jensen D, Gundestrup N.S., Hammer C.U., Hvidberg C.S., Steffensen J.P., SVEInbJÖrnSDÓtTIR A.E., Jouvel J., Bond G.C., 1993 : « Evidence for general instability of past climate from $250 \mathrm{kyr}$ ice-core record », Nature, 264, p. 218-220.

Delpech F., 1988 : «Les réponses des Ongulés du Pléistocène Supérieur aux changements climatiques en Aquitaine (Sud-Ouest de la France) Quelques exemples », Geobios, 21/4, p. 495-503.

Delpech F., 1999 : « Biomasse d'Ongulés au Paléolithique et inférences sur la démographie ", Paléo, 11, p. 19-42.

Demars P.-Y., 1996 : « Démographie et occupation de l'espace au Paléolithique supérieur et au Mésolithique en France », Préhistoire Européenne, 8, p. 3-26.

Demars P.-Y., 2000 : « Altitude des sites suivant le climat au Paléolithique supérieur et au Mésolithique en France », Praehistoria, 1, p. 47-54

Demars P.-Y., 2002a : «À la conquête des massifs montagneux. L'occupation de l'espace à la fin de la dernière glaciation et au début du Postglaciaire dans le Sud de la France », Préhistoire du Sud-Ouest, 9/2, p. $185-190$

Demars P.-Y., 2002b : « Changements climatiques et occupation de l'espace; Les derniers chasseurs-cueilleurs d'Europe face à la déglaciation ", Quaternaire, 13(3-4), p. 289-286.

Demars P.-Y., 2004 : «L'occupation du versant nord des Pyrénées : le rôle du climat », Préhistoire du Sud-Ouest, 11, p. 39-48.

Demars P.-Y., 2006 : « L'occupation de l'Europe par les chasseurs du Paléolithique supérieur : une question de climat ",M@ppemonde, 83 , $12 \mathrm{p}$. http://mappemonde.mgm.fr/num11/articles/arto60306.html

Demars P.-Y., 2008 : « Paléogéographie des chasseurs de l'Europe du Paléolithique supérieur : répartition et spécialisation des sites », L'Anthropologie, 112, p. 157-167.

Deschamps M., 2008: Le Vasconien et sa signification au sein des faciès moustériens. mémoire de Master 2, Université de Toulouse, 55 p.

Foucher P., SAN JuAN C., 2002 : "Considérations générales sur le Solutréen des Pyrénées : typologie et circulation des matières premières. Préhistoire Art et Sociétés », Bulletin de la Société Préhistorique Ariège-Pyrénées, LVII, p. 105-112.

FOUCHER P., 2004: Les industries lithiques du complexe GravettienSolutréen dans les Pyrénées. Techno-typologie et circulation des matières siliceuses de part et d'autre de l'axe Pyrénées-Cantabres, thèse de Doctorat, Université de Toulouse, $334 \mathrm{p}$.

FOUCHER P., SAN JUAN C., 2005. « La circulation des matières premières siliceuses dans le Gravettien pyrénéen », in JAUBERT, J., BARBAZA, M. (ÉDS), Territoires, déplacements, mobilités, échanges durant la Préhistoire. Terres et hommes du Sud, Paris, CTHS, p. 199-217.

Foucher P., San Juan C., C., SaCChi D., Arrizabalaga A., 2008 : «Le Gravettien des Pyrénées », Paléo, 2o, p. 331-356.

Fritz C., Tosello G., 2005 : « Entre Périgord et Cantabres: les magdaléniens de Marsoulas », in JAUbert, J., BARBAZA, M. (ÉDs), Territoires, déplacements, mobilités, échanges durant la Préhistoire. Terres et hommes du Sud, Paris, CTHS, p. 311-328.

GaILlaRd C., 1979 : L'industrie lithique du Paléolithique inférieur et moyen de la grotte de Coupe-Gorge à Montmaurin (Haute-Garonne), thèse de Doctorat, Université de Provence, $586 \mathrm{p}$.

Gamble C. S., 1986 : The Palaeolithic settlement of Europe, Cambridge Cambridge University Press, $471 \mathrm{p}$

Gamble C. S., Davies W., Pettit P., Richards M., 2004 : « Climate change and evolving human diversity in Europe during the last glacial », in Willis K.J., BenNett K.D., WALKer D. (ÉDS.), The Evolutionary Legacy of the Ice Ages, Philosophical Transactions of the Royal Society Biological Sciences, 359, 243-254.

HubSCHMAN J., 1975a : Morphogenèse et pédogenèse quaternaires dans le piémont des Pyrénées garonnaises et ariégoises, thèse de Doctorat, Université de Toulouse, Le Mirail, Paris, Honoré Champion éd., 745 p.

HuBSCHMAN J., 1975b : Terrefort molassique et terrasses récentes de la région toulousaine. in HUBSCHMAN, J. (DIR.), Le piémont quaternaire de la Garonne (sud-est Aquitain, piémont central nord-pyrénéen), Bulletin de l'Association Française pour l'étude du Quaternaire, 44-45, p. 125-136.

ICOLE M., 1973 : Géochimie des altérations dans les nappes d'alluvions du piémont pyrénéen. thèse de Doctorat, Université de Paris XI, $200 \mathrm{p}$.

JARRY M., 2008 : «L'archéologie paléolithique à la reconquête de territoires oubliés ", Archéopages, hors série (Construction(s) de l'archéologie), p. 28-33.

JARRY M., 2010: Les groupes humains du Pléistocène moyen et supérieur en Midi toulousain: contextes, ressources et comportements entre Massif Central et Pyrénées, thèse de Doctorat, Université de Toulouse, $470 \mathrm{p}$

JAUBert J., 2002 : « Le Paléolithique dans le Midi toulousain », in PAILleR J.-M. (DIR.), Tolosa, nouvelles recherches sur Toulouse et son histoire dans l'Antiquité, École Française de Rome, 281, p. 43-52.

KLARIC L 2008 : " Anciennes et nouvelles hypothèses d'interprétation du Gravettien moyen en France : la question de la place des industries à burins du Raysse au sein de la mosaïque gravettienne », Paléo, 20, p. 257-276.

Klaric L., Guillermin P., Aubry T., 2009: « Des armatures variées et des modes de productions variables. Réflexions à partir de quelques exemples issus du Gravettien d'Europe occidentale (France, Portugal, Allemagne) », Gallia Préhistoire, 51, p. 113-154.

Klingebiel A., Legigan P., 1985 : « Histoire géologique de la Grande Lande ", in La Grande Lande: histoire naturelle et géographie historique, Actes du colloque, Sabres, 27 novembre 1981, p. 13-30.

Le Brun-Ricalens F., 1988 : Contribution à l'étude du Paléolithique du Pays de Serre du Bas Quercy et de l'Agenais entre les vallées du Lot et de la Garonne, mémoire de DEA, Université de Toulouse le Mirail, $452 \mathrm{p}$.

Le Brun-Ricalens F., Séronie-Vivien M.-R., 2004 : « Présence d'un silex d'origine nord-pyrénéenne (Chalosse?) en Haut-Quercy dans l'Aurignacien du Piage (Lot, France) et implications », Paléo, 19, p. 341-366.

LEROI-GOURHAN A., 1981 : « Les signes pariétaux comme marqueurs ethniques », Altamira Symposium 1979, Actes du symposium, Madrid Asturias-Santender, Madrid, p. 289-294.

Lelouvier L.-A., Bosc-Zanardo, B., Bruxelles L., Chalard P., JARRY M., à paraître : « La station de plein air d'En-Vignes : indices d'une occupation magdalénienne, Marsan (Gers) », Bulletin de la Société Préhistorique Française.

MÉroc L., 1961 : « Le remplissage des cavernes des Pyrénées : essai sur l'évolution du climat pyrénéen durant le Quaternaire », Symposium international de spéléologie, Coma, p. 2-13.

Méroc L. 1963 : «L'Aurignacien et le Périgordien dans les Pyrénées françaises et dans leur avant-pays », Bulletin de la Société Méridionale de Spéléologie et de Préhistoire, VI-IX (1956-9), p. 63-74.

Mourre V., Castamagno S., Thiebault, C., Allard M., Jeannet M., Juillard F., Bruxelles L., Colonge D., Cravinho S. Laroulandie V., Maureille B., 2007 : «Le site moustérien de la Grotte du Noisetier à Fréchet-Aure (Hautes-Pyrénées) : premiers résultats des nouvelles fouilles », in JAUbert J., Bordes J.-G., Ortega I. (ÉDS.), Les Sociétés du Paléolithique dans le Grand-SudOuest de la France: nouveaux gisements, nouveaux résultats, nouvelles méthodes, Journées SPF, Université de Bordeaux 1, Talence, 24-25 nov. 2006, Société Préhistorique Française (mémoires, XLVII), p. 189-202.

Отте M., 1983 : «Le Paléolithique de Belgique. Essai de synthèse », L'Anthropologie, 87/3, p. 291-321.

Pallas R., Rodes A., Braucher R., Carcaillet J., Ortuno M., Bordonau J., Boules D., Vilaplana J.M., Masana E., SANTANACH P., 2006 : « Late Pleistocene and Holocene glaciation in the Pyrenees: a critical review and new evidence from $10 B e$ exposure ages, south-central Pyrenees », Quaternary Science Review, 25, p. 2937-2963.

Peltier W.R., 1994 : «Ice age paleotopography », Science, 265, p. 195-201.

Pessese D., 2008: « Le statut de la fléchette au sein des premières industries gravettiennes ", Paléo, 20, p. 277-290.

Pinos A., 1999: L'Homme de Neandertal dans le Midi-toulousain: approche typologique et technologique de deux séries lithiques issues de stations de plein air, Archéo en Savès, Archéologie, Histoire et Ethnographie en Gascogne orientale, $101 \mathrm{p}$.

Roebroeks W., Conard N. J., Van Kolfschoten T., 1992 : « Dense forests, cold steppes, and the palaeolithic settlement of Northern Europe », Current Anthropology, 33/5, p. 551-586.

Revel J.-C., Bourgeat F., PaQuet H., 1978 : « Pédogenèse quaternaire dans la région toulousaine : les lœss et leurs colluvions comme marqueur chronologique ", Bulletin de l'Association Française pour l'Étude du Quaternaire 4, p. 179-185.

SACCHI D., 1986 : Le Paléolithique supérieur du Languedoc occidental et du Roussillon, Gallia Préhistoire (suppl. 21), Paris, CNRS, 284 p.

SENTIS J., 2005: «Les silhouettes féminines stylisées peuvent-elles caractériser des territoires culturels », in JAUBERT J., BARBAZA M. (ÉDS.), Territoires, déplacements, mobilités, échanges durant la Préhistoire. Terres et hommes du Sud, Paris, CTHS, p. 411-420.

Séronie-Vivein M., Séronie-Vivein M.-R., Foucher P., 2006 : «L'économie du silex au Paléolithique supérieur dans le Bassin d'Aquitaine. Le cas des silex à lépidorbitoïdes des Pyrénées centrales. Caractérisation et implications méthodologiques », Paléo, 18, p. 193-216.

SÉrONIE-VIVEIN M.-R., 2009: « Répartition géographique des différents types de silex à lépidorbitoïdes utilisés au Paléolithique supérieur en Aquitaine. Première contribution : Dordogne (Caminade), Lot (Piage-Pégourié-Cloup de l'Aze), Gironde (Beauregard) », Bulletin de la Société Préhistorique Française, 106/2, 293-396.

Simonnet R., 1996 : « Approvisionnement en silex au Paléolithique supérieur. Déplacements et caractéristiques physionomiques des paysages : l'exemple des Pyrénées centrales ", in Delporte H., Clottes J., (ÉDs.), Pyrénées préhistoriques, Arts et Sociétés, Paris, CTHS, p. 117-128.

Simonnet R., 1998 : « Le silex et les fins du Paléolithique supérieur dans le Bassin de Tarascon-sur-Ariège », Bulletin de la Société Préhistorique Ariège Pyrénées, LVII, p. 113-170.

Simonnet R., 2007: « Entre Pyrénées et Poitou-Berry vers 14 ooo : entre approvisionnement et/ou communication. Préhistoire, Art et Sociétés », Société Préhistorique Ariège-Pyrénées, LXII, p. 57-62. 
SORESSI M., 2002 : Le Moustérien de tradition acheuléenne dans le Sud-Ouest de la France. Discussion sur la signification du faciès à partir de l'étude comparée de quatre sites : Pech de l'Azé I, le Moustier, la Rochette et la Grotte XVI, thèse de Doctorat, Université de Bordeaux, $330 \mathrm{p}$.

Soressi M., Rendu W., Texier J.-P., Claud E., Daulny L., D'errico F, Laroulandie V., Maureille B., Niclot M., Schwortz S. Tillier A.-M., 2008 : « Pech-de-L'Azé I (Dordogne, France) : nouveau regard sur un gisement moustérien de tradition acheuléenne connu depuis le XIX ${ }^{e}$ siècle », in JAUBERT J., BORDES J.-G., Ortega I. (ÉDs.), Les Sociétés du Paléolithique dans le GrandSud-Ouest de la France: nouveaux gisements, nouveaux résultats, nouvelles méthodes, Journées SPF, Université de Bordeaux 1, Talence, 24-25 nov. 2006, Société Préhistorique Française (mémoires, XLVII), p. $95-132$.

SORIANO S., 2005: « Le Sud : une plate-forme pour le peuplement des espaces septentrionaux », in JAUBERT J., BARBAZA M. (DIR.), Territoires, déplacements, mobilités, échanges durant la Préhistoire. Terres et hommes du Sud, Actes des congrès nationaux des sociétés historiques et scientifiques, Toulouse, 2001, Paris, CTHS, p. 63-83.

Straus L., 1991 : « Human geography of the late Upper Paleolithic in western Europe », Journal of Anthropological Research, 47, p. 259-278

Tavoso A., 1986 : Le Paléolithique inférieur et moyen du HautLanguedoc. Gisements des terrasses alluviales du Tarn, du Dadou, de l'Agout, du Sor et du Fresquel, université de Provence (Études Quaternaires, 5), éd. du Laboratoire de Paléontologie Humaine et de Préhistoire, 404 p.

Tuffreau A., 1992 : «Middle paleolithic settlement in Northern France», in Dibble H.L., Mellars P. (ÉDS.), The Midlle Paleolithic: adaptation, behavior and variability, University Museum symposium (series 2), Philadelphia: University of Pennsylvania, p. 6o-73.

Turq A., Brenet M., Colonge D., Jarry M., Lelouvier L.-A., O'FARrel M., JAUbert J., 2010: « Premières occupations humaines dans le Sud-Ouest de la France : un bilan réactualisé, vingt ans après le colloque d'Abbeville/Saint-Riquier ", Quaternary International, 223/224, p. 383-398

VANHAeren M., D'ERrico F., 2006 : « Aurignacian ethno-linguistic geography of Europe revealed by personal ornaments », Journal of Archaeological Science, 33, p. 1105-1128.

VIALOU D., 1986 : L'art des grottes en Ariège magdalénienne, Gallia Préhistoire (suppl. 22),Paris, CNRS, 425 p.

\section{Des tombes chasséennes attestées dans le Toulousain}

\author{
Fabrice Pons \\ Inrap, UMR 5140 \\ Muriel Gandelin \\ Inrap, UMR 5608, Travaux et Rercherches Archéologiques \\ sur les Espaces, les Cultures et les Sociétés, MSH Toulouse. \\ Jérôme Rouquet \\ Inrap
}

J usquà récemment, la quasi-totalité des sépultures chasséennes découvertes dans le Toulousain, 25 au total, provenait de structures dont la fonction initiale n'était pas funéraire. Près de 50 ans après la première sépulture chasséenne fouillée dans le Midi de la France par L. Méroc à VilleneuveTolosane (2008), une aire d'inhumation a enfin été mise au jour lors d'une vaste opération de fouille préventive à Sauzas (Blagnac, Haute-Garonne). Cet ensemble, reconnu sur environ $150 \mathrm{~m}^{2}$, dans un secteur où n'avait été mis en évidence aucun élément de cette période, rassemble six tombes situées à l'écart des zones d'habitats néolithiques et totalement insoupçonnées [Fig.1].

Des tombes sous tertre? Les tombes identifiées ne se recoupent pas. Quatre sont distantes de 1 à $3 \mathrm{~m}$, installées en enfilade selon un axe grossièrement orienté est-ouest. Les deux autres sont implantées plus au sud, à moins d'une dizaine de mètres des précédentes pour la sépulture la plus méridionale [Fig.2]. D'après l'emprise de la fouille, il n'est pas impossible, que l'aire funéraire se développe encore plus au sud de cette dernière. En revanche, les décapages réalisés vers l'ouest et le nord n'ont pas fourni les indices d'une extension de la zone funéraire dans ces directions.

Les sépultures les mieux documentées se caractérisent par une structure formée d'un amas, parfois assez dense, de galets. Prélevés sur les berges de la Garonne, ceux-ci sont d'un module constant, indice probable d'une certaine sélection. Ils recouvrent directement la quasi-totalité du squelette et une partie du mobilier d'accompagnement [Fig.3]. L'absence de sédimentation importante laisserait supposer qu'ils ont été déposés directement sur le corps du défunt. Mais l'écrasement de certains vases d'accompagnement, provoqué par l'affaissement des galets, s'explique seulement par la présence de vides à l'intérieur du loculus après la fermeture de la tombe, hypothèse en partie corroborée par les déplacements importants de certaines pièces squelettiques. Cette couverture ne pouvait être maintenue au-dessus du dépôt funéraire qu'à l'aide d'une armature de soutien probablement réalisée en matériaux périssables (platelage de bois, coffrage...) qui en assurait la fermeture effective. La disposition des galets suggère également l'existence d'un léger creusement avec des effets de paroi nettement visibles. De plan sensiblement rectangulaire, cette fosse sépulcrale ne devait pas excéder une vingtaine de centimètres de profondeur.

Des inhumations dans la tradition du Chasséen méridional. Les inhumations présentent une grande homogénéité. Le corps du défunt est orienté selon un axe sud-nord, tête au sud. Il repose systématiquement sur le côté gauche, membres supérieurs et inférieurs fléchis en un semblant de position fotale [Fig.4]. Cette position est très majoritaire dans le Toulousain (19 cas sur 26 documentés) et quasi exclusive chez les adultes puisque 16 sur les 20 recensés adoptent cette position.

Lorsqu'il a pu être caractérisé, l'espace de décomposition s'est toujours révélé vide. Ces inhumations sont toutes des sépultures individuelles de sujets adultes, sauf une qui a livré les restes de deux individus, un homme et un enfant (âgé de 1 à 4 ans) [Fig.5]. Le statut de ce dernier soulève des interrogations dans la mesure où il semble avoir été déposé en même temps et au même endroit que le mobilier d'accompagnement du sujet adulte. Il enrichit le débat sur les morts d'accompagnement au sein des sépultures chasséennes.

\section{Un riche mobilier d'accompagnement.}

Toutes les sépultures comprennent du mobilier d'accompagnement, dans lequel il demeure difficile de distinguer, d'un côté, celui qui a été déposé «en offrande» afin d'accompagner le défunt dans l'au-delà («viatique») et, d'un autre côté, celui qui provient des attributs personnels du défunt.

La présence de vases s'avère systématique. Ces objets ont sans doute été déposés entiers même si, dans la plupart des cas, une partie seulement se trouve conservée. Ils semblent avoir été placés en position fonctionnelle comme peuvent le suggérer les récipients destinés à recevoir des offrandes 